Gut and Liver, Vol. 9, No. 5, September 2015, pp. 615-622

\title{
Clinical and Endoscopic Features of Metastatic Tumors in the Stomach
}

Ga Hee Kim*, Ji Yong Ahn*, Hwoon-Yong Jung*, Young Soo Park ${ }^{\dagger}$, Min-Ju Kim ${ }^{\ddagger}$, Kee Don Choi*, Jeong Hoon Lee*, KwiSook Choi*, Do Hoon Kim*, Hyun Lim*, Ho June Song*, Gin Hyug Lee*, and Jin-Ho Kim*

Departments of *Gastroenterology, ${ }^{\dagger}$ Pathology, and ${ }^{\ddagger}$ Clinical Epidemiology and Biostatistics, Asan Medical Center, University of Ulsan College of Medicine, and Asan Digestive Disease Research Institute, Seoul, Korea

Background/Aims: Metastasis to the stomach is rare. The aim of this study was to describe and analyze the clinical outcomes of cancers that metastasized to the stomach. Methods: We reviewed the clinicopathological aspects of patients with gastric metastases from solid organ tumors. Thirty-seven cases were identified, and we evaluated the histology, initial presentation, imaging findings, lesion locations, treatment courses, and overall patient survival. Results: Endoscopic findings indicated that solitary lesions presented more frequently than multiple lesions and submucosal tumor-like tumors were the most common appearance. Malignant melanoma was the tumor that most frequently metastasized to the stomach. Twelve patients received treatments after the diagnosis of gastric metastasis. The median survival period from the diagnosis of gastric metastasis was 3.0 months (interquartile range, 1.0 to 11.0 months). Patients with solitary lesions and patients who received any treatments survived longer after the diagnosis of metastatic cancer than patients with multiple lesions and patients who did not any receive any treatments. Conclusions: Proper treatment with careful consideration of the primary tumor characteristics can increase the survival period in patients with tumors that metastasize to the stomach, especially in cases with solitary metastatic lesions in endoscopic findings. (Gut Liver 2015;9:615-622)

Key Words: Stomach; Endoscopy; Metastasis

\section{INTRODUCTION}

The presence of metastasis in the stomach is rare, with a reported incidence of $0.2 \%$ to $1.7 \%$ according to clinical and autopsy findings. ${ }^{1,2}$ Previous studies report that lung, breast, melanoma, and esophageal carcinomas can metastasize to the stomach, even though the risk is very low. ${ }^{3,4}$ The mechanisms underlying gastric metastasis have not been clearly elucidated and are probably different for every primary tumor. Four pathways may be involved in the metastatic spread of the original primary cancers to the stomach: peritoneal dissemination, hematogenous dissemination, lymphatic spread, and direct tumor invasion. ${ }^{5}$

Generally, patient prognosis and survival outcome are poor because the presence of gastric metastasis is associated with advanced disease. ${ }^{6}$ However, there are very few case reports on metastatic cancer in the stomach due to their low incidence. ${ }^{7,8}$ Clinical outcomes of metastatic tumor to the stomach are not reported yet. In this study, we gathered and reviewed the data on the patients with metastasis in the stomach in order to show the clinicopathologic features, clinical outcomes and prognostic factors.

\section{MATERIALS AND METHODS}

\section{Patients}

Between January 1995 and December 2012, a total of 26,424 patients with gastric malignancy visited Asan Medical Center. Patients without endoscopic findings in their medical records or histological confirmation, and patients with leukemia, malignant lymphoma, or direct invasion from adjacent organs, were excluded from the analysis. Finally, there was a total of 37 patients diagnosed with metatstatic tumors in the stomach. The medical records of these patients were retrospectively reviewed. This study was approved by the Institutional Review Board of Asan Medical Center.

\section{Correspondence to: Hwoon-Yong Jung}

Department of Gastroenterology, Asan Medical Center, University of Ulsan College of Medicine, and Asan Digestive Disease Research Institute, 88 Olimpic-ro 43-gil, Songpa-gu, Seoul 138-736, Korea

Tel: +82-2-3010-3197, Fax: +82-2-476-0824, E-mail: hyjung@amc.seoul.kr

Received on January 27, 2014. Revised on April 28, 2014. Accepted on June 9, 2014. Published online December 5, 2014

pISSN 1976-2283 eISSN 2005-1212 http://dx.doi.org/10.5009/gnl14032

Ga Hee Kim and Ji Yong Ahn contributed equally to this work as first authors.

@ This is an Open Access article distributed under the terms of the Creative Commons Attribution Non-Commercial License (http://creativecommons.org/licenses/by-nc/4.0) which permits unrestricted non-commercial use, distribution, and reproduction in any medium, provided the original work is properly cited. 


\section{Endoscopic patterns of metastatic lesions in the stomach}

Esophagogastroduodenoscopy (EGD) was used for the diagnosis, and all the lesions were confirmed using endoscopic biopsy followed by histological analysis. Collected data included epidemiological characteristics, symptomatology, indications for endoscopic investigation, macroscopic presentation, time between primary tumor diagnosis and the detection of gastric metastasis, and treatment. Endoscopic procedures were performed using a single-channel endoscope (GIF-H260; Olympus Optical Co., Ltd., Tokyo, Japan). Using standard biopsy forceps, biopsies were obtained from suspected lesions under direct-vision endoscopy. Two endoscopists (J.Y.A. and H.Y.J.) reviewed the endoscopic findings and categorized the gross findings into two main patterns: resembling submucosal tumor or resembling primary gastric cancer. Those resembling primary gastric cancer were subdivided into two groups: resembling early gastric cancer and resembling advanced gastric cancer (Fig. 1). Those resembling advanced gastric cancer were further subdivided into four types. Type 1 is a polypoid tumor. Type 2 is an ulcerated tumor with sharply demarcated margins. Type 3 is an ulcerated tumor without definite borders. Type 4 is diffusely infiltrating tumor.

\section{Histologic patterns of metastatic lesions in the stomach}

The histological results were reviewed by the pathologists. The biopsy specimens were carefully studied and compared with the histological features of the primary tumors.

\section{Statistical analysis}

Categorical variables were analyzed using the Fisher exact test. The Mann-Whitney U test was used for continuous variables. Continuous variables are presented as median values with the interquartile range (IQR), and the categorical variables are presented as numbers with percentages. The Kaplan-Meier method and log-rank test were used to evaluate differences in overall survival. All p-values are two-sided, and $\mathrm{p}<0.05$ was considered statistically significant. All statistical analyses were performed using SPSS version 21.0 (SPSS Inc., Chicago, IL, USA).

\section{RESULTS}

\section{Baseline characteristics of patients with metastatic tu-} mors in the stomach

The baseline characteristics of the patients are summarized in Table 1 . The study population consisted of 21 male and 16 fe-
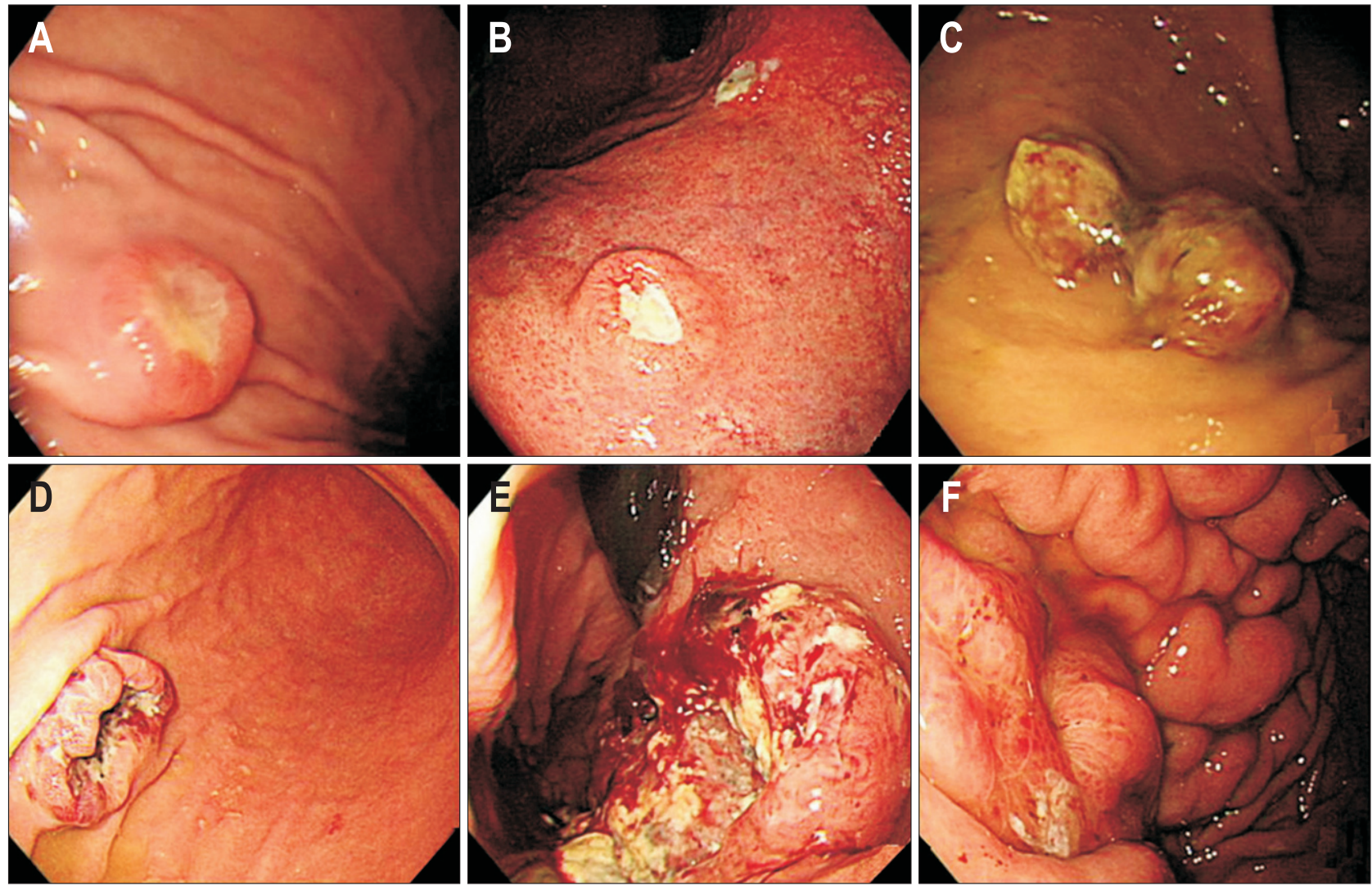

Fig. 1. Endoscopic appearance of metastatic tumors in the stomach. (A) Resembling submucosal tumors due to bladder cancer. (B) Resembling early primary gastric cancer due to lung adenocarcinoma. (C-F) Resembling advanced gastric cancer: (C) Type 1, due to renal cell carcinoma; (D) Type 2, due to choriocarcinoma; (E) Type 3, due to pulmonary squamous cell carcinoma; (F) Type 4, due to ovarian cancer. 
male patients (median age, 57.0 years; IQR, 49.5 to 61.0 years). The chief clinical manifestations of these 37 cases were gastrointestinal bleeding (12 cases, 32.4\%), abdominal pain (6 cases, 16.2\%), dysphagia (3 cases, 8.1\%), and vomiting ( 2 cases, 5.4\%). Another 11 patients had no symptoms. The endoscopic findings showed that solitary metastases (23 cases, 62.2\%) were more common than multiple metastases (14 cases, 37.8\%). The median interval between the diagnosis of the primary tumor and the diagnosis of the metastatic tumor in the stomach was 13.5 months (IQR, 0.75 to 33.5 months).

\section{Sites of the primary tumors}

As indicated in Table 2, the most common primary malig-

Table 1. Baseline Characteristics of the Study Patients with Metastatic Tumors in the Stomach $(\mathrm{N}=37)$

\begin{tabular}{|c|c|}
\hline Variable & Value \\
\hline \multicolumn{2}{|l|}{ Sex } \\
\hline Male & $21(56.8)$ \\
\hline Female & $16(43.2)$ \\
\hline Age, yr & $57.0(49.5-61.0)$ \\
\hline \multicolumn{2}{|l|}{ ECOG performance status } \\
\hline 0 & $3(8.1)$ \\
\hline 1 & $11(29.7)$ \\
\hline$\geq 2$ & $23(62.2)$ \\
\hline \multicolumn{2}{|l|}{ Main symptom } \\
\hline Gastrointestinal bleeding & $12(32.4)$ \\
\hline Abdominal pain & $6(16.2)$ \\
\hline Dysphagia & $3(8.1)$ \\
\hline Vomiting & $2(5.4)$ \\
\hline None & $14(37.8)$ \\
\hline \multicolumn{2}{|l|}{ Location } \\
\hline Body & $22(59.5)$ \\
\hline Antrum & $3(8.1)$ \\
\hline Cardia & $4(10.8)$ \\
\hline Fundus & $5(13.5)$ \\
\hline Whole stomach & $3(8.1)$ \\
\hline \multicolumn{2}{|l|}{ No. of lesions } \\
\hline Solitary & $23(62.2)$ \\
\hline Multiple & $14(37.8)$ \\
\hline \multicolumn{2}{|l|}{ Histological type } \\
\hline Melanoma & $10(27.0)$ \\
\hline Adenocarcinoma & $9(24.3)$ \\
\hline Squamous cell carcinoma & $8(21.6)$ \\
\hline Renal cell carcinoma & $3(8.1)$ \\
\hline Small cell carcinoma & $2(5.4)$ \\
\hline Other & $5(13.5)$ \\
\hline
\end{tabular}

Data represent the number of patients (\%) or median (interquartile range).

ECOG, Eastern Cooperative Oncology Group. nancy that lead to metastatic tumors in the stomach was malignant melanoma (10 cases, 27.0\%), followed by lung cancer (7 cases, 18.9\%), breast cancer (5 cases, 13.5\%), esophageal cancer (3 cases, 8.1\%), colorectal cancer (2 cases, 5.4\%), ovarian cancer (3 cases, 8.1\%), kidney cancer (3 cases, 8.1\%), head and neck cancer ( 1 case, $2.7 \%$ ), bladder cancer ( 1 case, $2.7 \%$ ), choriocarcinoma ( 1 case, $2.7 \%$ ), and neuroblastoma (1 case, 2.7\%). Among melanoma cases, the confirmed primary tumor site was the skin in 6 cases, but the primary site could not be determined in 4 cases. Among 7 lung cancers, 4 squamous cell carcinoma, 2 adenocarcinomas, and 1 epithelioid tumor were identified.

\section{Endoscopic appearance of metastatic tumors in the stomach}

Table 3 shows the endoscopic appearance of the metastatic tumors in the stomach, which were divided into two groups. The first group resembled submucosal tumors (12 cases, 32.5\%), and

Table 2. Site of the Primary Tumors in the Study Patients $(\mathrm{N}=37)$

\begin{tabular}{lc}
\hline \multicolumn{1}{c}{ Primary tumor } & No. (\%) \\
\hline Malignant melanoma & $10(27.0)$ \\
Lung & $7(18.9)$ \\
Breast & $5(13.5)$ \\
Gastrointestinal tract & \\
Esophagus & $3(8.1)$ \\
$\quad$ Colorectum & $2(5.4)$ \\
Genitourinary tract & \\
Ovary & $3(8.1)$ \\
Kidney & $3(8.1)$ \\
Bladder & $1(2.7)$ \\
Choriocarcinoma & $1(2.7)$ \\
Head/neck & $1(2.7)$ \\
Neuroblastoma & $1(2.7)$ \\
\hline
\end{tabular}

ECOG, Eastern Cooperative Oncology Group.

Table 3. Endoscopic Appearance of Metastatic Tumors in the Stomach $(\mathrm{N}=37)$

\begin{tabular}{ll}
\hline \multicolumn{1}{c}{ Endoscopic appearance } & No. (\%) \\
\hline $\begin{array}{l}\text { Resembling submucosal tumors } \\
\text { Resembling primary gastric cancer } \\
\text { Resembling early cancer }\end{array}$ & $12(32.4)$ \\
Resembling advanced cancer & $7(18.9)$ \\
Type 1 & \\
Type 2 & $7(18.9)$ \\
Type 3 & $2(5.4)$ \\
Type 4 & $5(13.5)$ \\
\hline
\end{tabular}

Type 1 , a polypoid tumor; Type 2 , an ulcerated tumor with sharply demarcated margins; Type 3, an ulcerated tumor without definite borders; Type 4 , diffusely infiltrating tumor. 


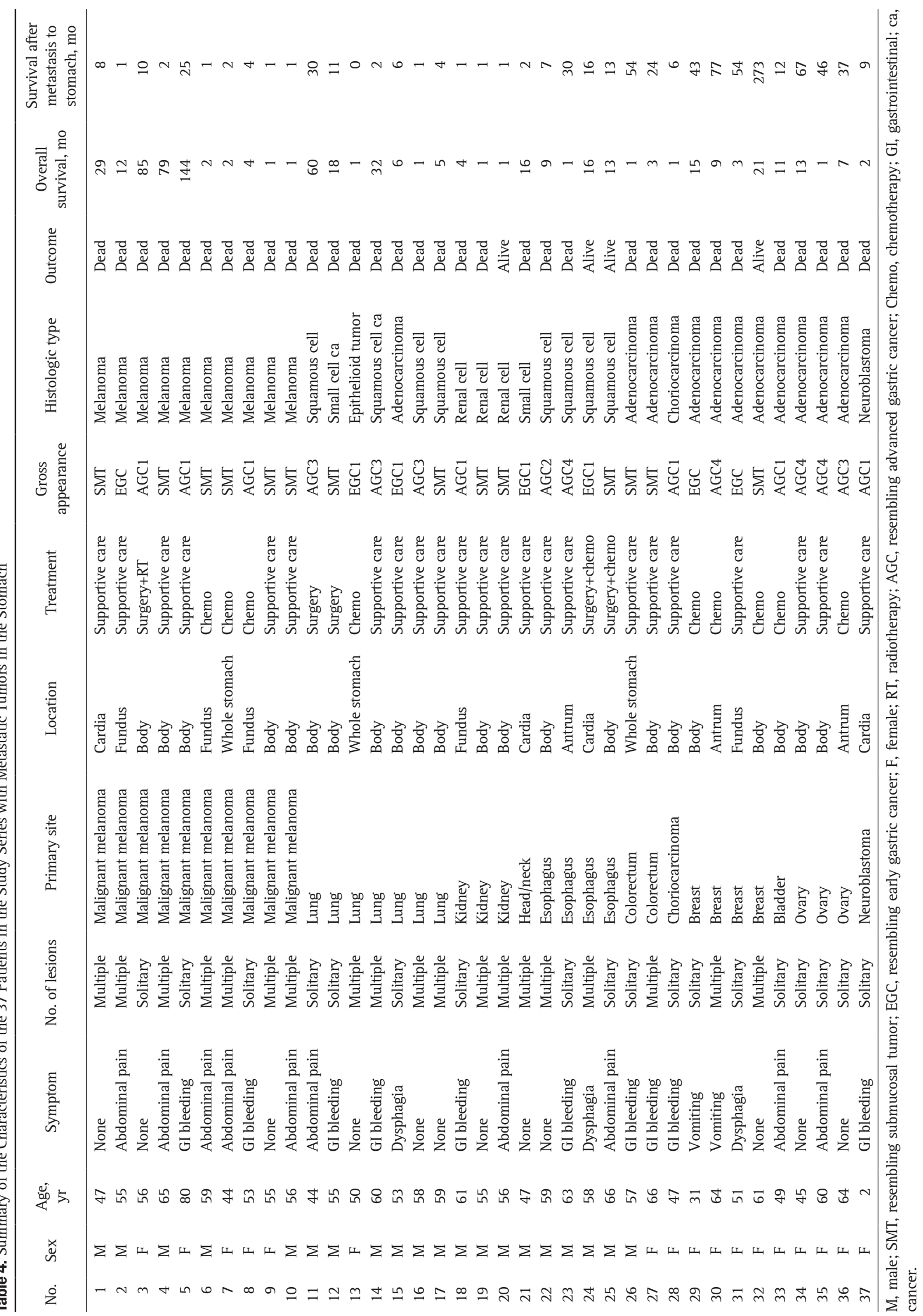


the second group (25 cases, 67.5\%) resembled primary gastric cancer. Among patients with metastasis that resembled early gastric cancer, 2 breast cancers, 2 lung cancers, 1 malignant melanoma, 1 head and neck cancer, and 1 esophageal cancer were identified. Among patients with metastasis that resembled advanced gastric cancer, 3 malignant melanomas, 3 lung cancers, 2 kidney cancers, 3 ovary cancers, 2 colorectal cancers, 2 esophageal cancers, 1 choriocarcinoma, 1 neuroblastoma, and 1

Table 5. Clinicopathologic Features of Metastatic Tumors in the Stomach $(\mathrm{N}=37)$

\begin{tabular}{lc}
\hline \multicolumn{1}{c}{ Clinicopathologic feature } & Value \\
\hline Treatment method of primary cancer & $2(5.4)$ \\
Surgery & $13(35.1)$ \\
Surgery+chemotherapy & $1(2.7)$ \\
Surgery+radiotherapy & $9(24.3)$ \\
Chemotherapy & $2(5.4)$ \\
Chemotherapy+radiotherapy & $1(2.7)$ \\
Radiotherapy & $5(13.5)$ \\
Conservative & \\
Treatment method of metastatic cancer & $2(5.4)$ \\
Surgery & $6(16.2)$ \\
Chemotherapy & $4(10.8)$ \\
Surgery+chemotherapy & $25(67.6)$ \\
Conservative & $33(89.2)$ \\
Dead & $4(10.8)$ \\
Alive & $18.0(6.0-18.0)$ \\
Survival after diagnosis of primary cancer, mo & $14.0(0.5-48.0)$ \\
Time span, mo* & $3.0(1.0-11.0)$ \\
Survival after diagnosis of metastatic cancer, mo & \\
\hline
\end{tabular}

Data represent the numbers of patients (\%) or median (interquartile range).

*Time between the diagnosis of the primary tumor and metastatic lesions.

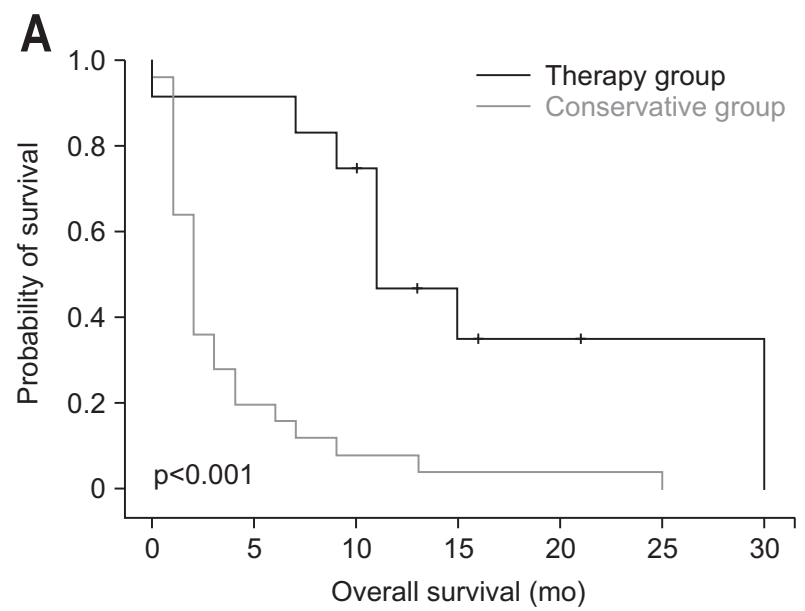

breast cancer were identified. Although gastric metastases may be recognized as abnormalities on EGD, there are no characteristic features that can be used to identify this disease because of the variable morphology of the tumors.

\section{Clinical outcomes of metastatic tumors in the stomach according to treatment}

The clinical data of the 37 study patients are summarized in Table 4. Only 12 patients received some form of treatment after the diagnosis of gastric metastasis. Surgical resection was performed on six patients. Partial gastrectomy for metastasis from lung cancer was performed on two patients, and wedge resection with chemotherapy was performed on one patient with metastatic malignant melanoma. Two patients with esophageal cancer and stomach metastasis underwent partial gastrectomy with concurrent chemoradiotherapy. One patient with renal cell carcinoma with stomach metastasis underwent wedge resection because of uncontrolled cancer bleeding. The median survival period of the 37 patients was 18.0 months (IQR, 6.0 to 18.0 months) after the diagnosis of primary cancer. Survival after the diagnosis of gastric metastasis was 3.0 months (IQR, 1.0 to 11.0 months) (Table 5).

Among the 12 patients who received treatment, the median survival period after the diagnosis of metastatic cancer and the median survival period after the diagnosis of primary cancer were longer than the 25 patients who did not receive treatment (11.0 months [IQR, 9.0 to 30.0 months] vs 2.0 months [IQR, 1.0 to 11.0 months, $\mathrm{p}<0.001$ ]; 60.0 months [IQR, 18.0 to 85.0 months] vs 14.0 months [IQR, 5.0 to 32.0 months], respectively; $\mathrm{p}=0.022$ ) (Fig. 2A).

\section{Clinical outcomes of solitary metastatic tumors and mul- tiple metastatic tumors in the stomach}

Among all 37 patients, solitary metastatic tumors in the stomach were found in 23 patients (62.2\%) and multiple lesions in 14 patients (37.8\%). Among patients with solitary metasta-

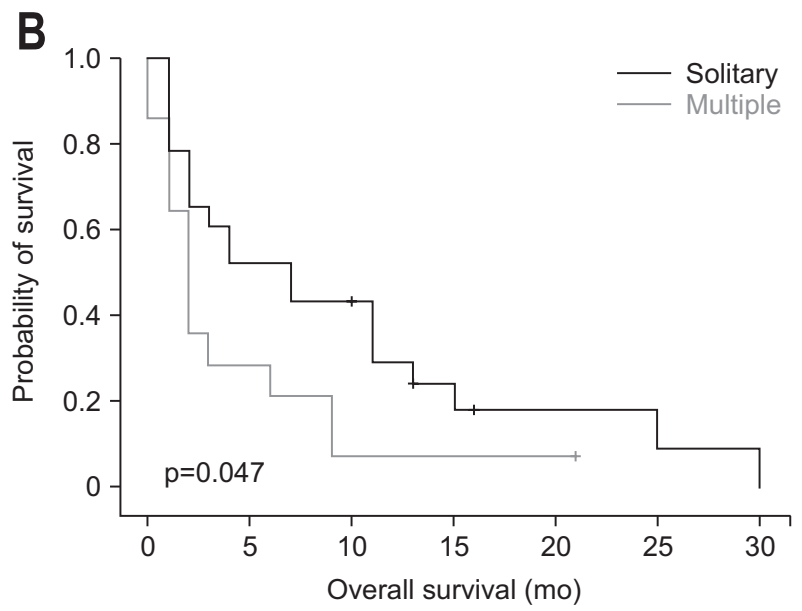

Fig. 2. Kaplan-Meier survival curves of (A) patients who underwent chemotherapy, surgery, or radiotherapy versus patients who underwent conservative treatment $(p<0.001)$; and (B) patients with solitary versus multiple tumors $(p=0.047)$. 
Table 6. Comparisons of the Clinical Features of Solitary and Multiple Lesions in the Study Cohort (N=37)

\begin{tabular}{|c|c|c|c|}
\hline Clinical feature & Solitary $(n=23)$ & Multiple $(\mathrm{n}=14)$ & p-value \\
\hline Sex & & & 0.471 \\
\hline Male & 12 & 9 & \\
\hline Female & 11 & 5 & \\
\hline Age, yr & $58.0(47.0-60.0)$ & $55.5(52.2-61.7)$ & 0.590 \\
\hline Site of primary tumor & & & 0.023 \\
\hline Melanoma & 3 & 7 & \\
\hline Nonmelanoma & 20 & 7 & \\
\hline Endoscopic appearance & & & 0.065 \\
\hline Resembling SMT & 4 & 7 & \\
\hline Resembling early cancer & 4 & 3 & \\
\hline Resembling advanced cancer & 15 & 4 & \\
\hline Treatment & 9 & 3 & 0.306 \\
\hline Death & 20 & 13 & 1.000 \\
\hline Time span, mo* & $28.0(1.0-51.0)$ & $12.0(0-38.5)$ & 0.412 \\
\hline Survival after diagnosis of primary tumor, mo & $32.0(9.0-54.0)$ & $13.0(2.0-30.0)$ & 0.445 \\
\hline Survival after diagnosis of metastatic tumor, mo & $7.0(2.0-13.0)$ & $2.0(1.0-6.0)$ & 0.047 \\
\hline
\end{tabular}

Data represent the numbers of patients or median (interquartile range).

SMT, resembling submucosal tumor.

*Time between the diagnosis of the primary tumor and metastatic lesions.

sis, six patients underwent gastrectomy. Four of these patients received systemic chemotherapy after surgery. Table 6 summarizes of the clinical factors in comparison with patients with a solitary lesion and patients with multiple lesions. Patients with a solitary lesion demonstrated longer median survival after the diagnosis of metastatic cancer than patients with multiple lesions (7.0 months [IQR, 2.0 to 13.0 months] vs 2.0 months [IQR, 1.0 to 6.0 months], as respectively; $\mathrm{p}=0.047$ ). Kaplan-Meier survival curves show that patients with a solitary lesion demonstrated better prognosis than patients with multiple lesions (Fig. 2B).

\section{DISCUSSION}

The stomach is an unusual site for metastasis. As a rare condition, the actual incidence of metastasis to the stomach is difficult to assess. Only $0.2 \%$ to $1.7 \%$ of cancer patients with metastatic disease reportedly develop metastasis in the stomach, and this diagnosis is typically made at autopsy. ${ }^{1,7}$ However, because the prognosis of cancer patients has gradually improved, gastric metastasis is encountered more frequently. Moreover, there has been not enough studies about the clinical outcomes about the metastatic tumor to the stomach, especially about the endoscopic features and the proper treatment to the patients. The study shows that patients with solitary lesions and patients who receive treatment demonstrate longer survival after the diagnosis of metastatic cancer than patients with multiple lesions and patients who have not received treatment (7 months vs 2 months [ $p=0.047]$ and 11 months vs 2 months $[p<0.001])$. Based on these results, administration of proper treatment to select patients, especially those with solitary metastatic lesions, can offer the chance of a longer term survival despite poor overall prognosis and heterogeneity of the primary tumors.

The management of gastric metastasis varies depending on the treatment method. Success has been documented using several approaches, including surgery, endoscopy, radiotherapy, chemotherapy, and hormonal therapy. ${ }^{8}$ Because the presence of gastric metastasis is associated with advanced disease, the survival period is extremely short and treatment for metastatic tumors in the stomach usually consists of systemic therapy rather than surgery. ${ }^{9}$ However, in patients with a solitary metachronous gastric metastasis, the surgical resection of metastatic gastric tumors may be recommended in order to control hemorrhage and solitary metastasis in the stomach may be removed using minimally invasive methods such as endoscopic mucosal resection. ${ }^{10,11}$ In the present study, surgical resection, such as partial gastrectomy or wedge resection, was performed on six patients. Surgical resection was performed on another six patients, partial gastrectomy of metastasis from lung cancer was performed on two patients, and wedge resection with chemotherapy was performed on one patient with metastatic malignant melanoma. Two patients with esophageal cancer and stomach metastasis received partial gastrectomy with concurrent chemoradiotherapy. One patient with renal cell carcinoma and stomach metastasis received wedge resection because of uncontrolled cancer bleeding. Interestingly, five of six patients who received surgery survived $>1$ year following treatment. 
Such outcomes may suggest that aggressive treatment strategies may benefit to patients who have with appropriate conditions for the treatment.

In a previous study, solitary metastases were reported to be more common than multiple metastases, and lesions were mainly located in the body of the stomach. ${ }^{3}$ In the present study, solitary metastases (23 cases, 62.2\%) were found to be more common than multiple metastases (14 cases, 37.8\%). Among our study patients with a solitary lesion, 14 cases (60.9\%) developed metastasis in the body of the stomach and nine patients (39.1\%) developed metastasis in another part of stomach. Of the patients with multiple lesions, eight (57.1\%) developed metastasis in the body of the stomach and six patients (42.9\%) developed metastasis in another part of stomach, thereby corroborating similar observations by Oda et al. ${ }^{3}$ However, there have been no studies that compared the clinical outcomes of solitary and multiple lesions. The present study shows that patients with a solitary lesion demonstrate a longer median survival after the diagnosis of metastatic cancer than patients with multiple lesions (7.0 months vs 2.0 months, $\mathrm{p}=0.047$ ). Therefore, in the absence of widely metastatic disease in patients with the proper conditions for surgery, aggressive therapy may offer the chance of longterm survival to patients with solitary metastasis.

Most patients with metastatic tumors in the stomach are asymptomatic. The symptoms of metastatic tumors, including pain, nausea, vomiting, and signs of bleeding, are nonspecific. ${ }^{12}$ Iron-deficiency anemia and a guaiac-positive stool may also present. ${ }^{13}$ In a previous autopsy series, abdominal pain was the most common symptom followed by nausea, vomiting, anorexia, and acute upper gastrointestinal bleeding. The current study shows that gastrointestinal bleeding (12 cases, 32.4\%) is the most common presentation, and endoscopy is an important diagnostic tool for evaluating metastatic lesions as well as evaluating the results of therapeutic intervention. Accordingly, we believe that endoscopic examination and histologic evaluation should be performed to identify any metastatic gastrointestinal lesions in patients with known primary cancer and symptoms related to gastrointestinal tracts, especially abdominal pain and gastrointestinal bleeding.

In metastatic cancers to the stomach, three main morphological features of endoscopic findings have been reported: nonulcerative masses, submucosal tumor masses with elevation and ulceration at the apex (volcano lesions), and multiple nodules of varying sizes with tip ulceration. ${ }^{14}$ Rarely, some lesions may appear as raised plaques, fat ulcers, or thickened walls. In our present study, submucosal tumors and polypoid masses were common endoscopic features. This finding is considered to be related with hematogenous dissemination and lymphatic spread of the primary tumor through the submucosal layer being the main metastatic pathway. Therefore, when submucosal tumors and polypoid masses are found in patients with malignancy, thorough endoscopic scrutinzation with biopsies and careful history taking should be conducted to improve diagnostic accuracy of gastric metastasis.

The mechanisms underlying gastric metastasis have not been clearly elucidated and are most likely different for each primary tumor. There are four pathways that may be involved in the metastatic spread of original primary cancers to the stomach: peritoneal dissemination, hematogenous dissemination, lymphatic spread, and direct tumor invasion. ${ }^{5}$ Previous studies reveal that the breast and lung are the most common primary sites, which may reflect the high incidences of these tumors in the general population. ${ }^{3}$ In malignant melanoma, the incidence of gastric metastasis is high because of the high tropism of the tumor to the gastrointestinal tract. According to the literature, about half of all patients with gastric metastasis concomitantly demonstrate metastatic lesions in other organs and the mean time from the diagnosis of gastric metastasis to death is approximately 4.75 months. ${ }^{15}$ In the present study, the most common primary lesion was found to be melanoma followed by lung cancer (7 cases, 18.9\%), breast cancer (5 cases, 13.5\%), esophageal cancer (3 cases, 8.1\%), colorectal cancer (2 cases, 5.4\%), ovarian cancer ( 3 cases, 8.1\%), and kidney cancer (3 cases, 8.1\%). Similar results have been reported in an earlier study in which the majority of the primary tumors included breast carcinoma, melanoma, and lung neoplasm. ${ }^{3}$ of these, half of metastatic tumors were found within a year of the diagnosis of the primary tumors (14.0 months [IQR, 0.5 to 48.0 months]). Endoscopic examinations should be carefully conducted and gastric metastasis should be considered for patients with melanoma, lung cancer, or breast cancer, especially when symptoms are evident.

The current study has limitations due to its retrospective design and small population size. The primary malignancies and treatments were also heterogenous. Furthermore, comparisons of clinical outcomes due to treatment and supportive care are limited because of differences in the medical conditions of the patients in both groups. Nevertheless, the current study will provide an impetus for future studies on the outcomes associated with metastatic cancers in the stomach.

In conclusion, metastatic tumors in the stomach may originate from various organs and show poor prognosis. Patients with solitary lesions and patients who had received any treatments survived longer after the diagnosis of metastatic cancer than patients with multiple lesions and patients who did not receive any treatments. Therefore, proper treatment with careful consideration about the characteristics of the primary tumors can increase the survival period in patients with metastatic tumor to the stomach, especially in cases with solitary metastatic lesions in endoscopic findings.

\section{CONFLICTS OF INTEREST}

No potential conflict of interest relevant to this article was reported. 


\section{REFERENCES}

1. Menuck LS, Amberg JR. Metastatic disease involving the stomach. Am J Dig Dis 1975;20:903-913.

2. Kadakia SC, Parker A, Canales L. Metastatic tumors to the upper gastrointestinal tract: endoscopic experience. Am J Gastroenterol 1992;87:1418-1423.

3. Oda, Kondo H, Yamao T, et al. Metastatic tumors to the stomach: analysis of 54 patients diagnosed at endoscopy and 347 autopsy cases. Endoscopy 2001;33:507-510.

4. Saito T, Iizuka T, Kato H, Watanabe H. Esophageal carcinoma metastatic to the stomach: a clinicopathologic study of 35 cases. Cancer 1985;56:2235-2241.

5. Namikawa T, Hanazaki K. Clinicopathological features and treatment outcomes of metastatic tumors in the stomach. Surg Today 2014;44:1392-1399.

6. De Palma GD, Masone S, Rega M, et al. Metastatic tumors to the stomach: clinical and endoscopic features. World J Gastroenterol 2006;12:7326-7328.

7. Green LK. Hematogenous metastases to the stomach: a review of 67 cases. Cancer 1990;65:1596-1600.

8. Taal BG, den Hartog Jager FC, Steinmetz R, Peterse H. The spectrum of gastrointestinal metastases of breast carcinoma: I. stom- ach. Gastrointest Endosc 1992;38:130-135.

9. Taal BG, Peterse H, Boot H. Clinical presentation, endoscopic features, and treatment of gastric metastases from breast carcinoma. Cancer 2000;89:2214-2221.

10. Siriwardana HP, Harvey MH, Kadirkamanathan SS, Tang B, Kamel D, Radzioch R. Endoscopic mucosal resection of a solitary metastatic tumor in the stomach: a case report. Surg Laparosc Endosc Percutan Tech 2012;22:e132-e134.

11. Kim MY, Jung HY, Choi KD, et al. Solitary synchronous metastatic gastric cancer arising from T1b renal cell carcinoma: a case report and systematic review. Gut Liver 2012;6:388-394.

12. Kim YI, Kang BC, Sung SH. Surgically resected gastric metastasis of pulmonary squamous cell carcinoma. World J Gastrointest Surg 2013;5:278-281.

13. Reiman T, Butts CA. Upper gastrointestinal bleeding as a metastatic manifestation of breast cancer: a case report and review of the literature. Can J Gastroenterol 2001;15:67-71.

14. Hsu CC, Chen JJ, Changchien CS. Endoscopic features of metastatic tumors in the upper gastrointestinal tract. Endoscopy 1996;28:249-253.

15. Campoli PM, Ejima FH, Cardoso DM, et al. Metastatic cancer to the stomach. Gastric Cancer 2006;9:19-25. 